\title{
Little bang at big Accelerators: Heavy ion physics from AGS to LHC
}

\author{
J. Schukraft \\ CERN, Geneva, Switzerland
}

\begin{abstract}
Since the start of ultra-relativistic heavy ion experimentation, some 10 years ago at the Brookhaven AGS and the CERN SPS, it has rarely been as gratifying to open a conference in this field as it is in Jaipur for this 3rd International Conference on Physics and Astrophysics of the Quark-Gluon-Plasma. The advent of a new generation of detectors, and most important, the availability of really heavy ion beams, has lead in the last three years to exciting new results which are of relevance to the most crucial questions this field has been addressing since 1986: are there signs for deconfinement, signs for chiral symmetry restoration, signs for equilibrium hadronic matter visible in ultra-relativistic heavy ion collisions? The tantalizing answer today to each of these questions seems to be: yes!

If the quest for the QGP is today in its most exciting and productive phase ever, the city of Jaipur is likewise a most pleasant and appropriate place to discuss the most recent progress. India has become a strong and recognized partner in heavy ion physics, with more than 40 experimentalists active in the CERN program and being the 5th largest country in ALICE, and with numerous and important contributions to theory. One can only congratulate the organizers of this conference for their excellent choice of time and place.

This opening summary will sketch a rough picture of the heavy ion program at current and future machines and concentrate on a few important topics, leaving the in-depth discussions to the specialized contributions following in these proceedings.
\end{abstract}

Opening talk of the 3rd International Conference on Physics and Astrophysics of the Quark-Gluon Plasma, March 17-21, Jaipur, India 
The aim of high-energy heavy-ion physics is the study of strongly interacting matter at extreme energy densities. Statistical QCD predicts that, at sufficiently high density, there will be a transition from hadronic matter to a plasma of deconfined quarks and gluons - a transition which in the early universe took place in the inverse direction some $10^{-5} \mathrm{~s}$ after the Big Bang and which might play a role still today in the core of collapsing neutron stars. The study of the phase diagram of nuclear matter (see Fig. 1), utilizing methods and concepts from both nuclear and high-energy physics, constitutes a new and interdisciplinary approach in investigating matter and its interactions. In highenergy physics, interactions are derived from first principles (gauge theories), and the matter concerned consists mostly of single particles (hadrons/quarks). In contrast, on nuclear physics scales the strong interaction is shielded and can therefore to date only be described in effective theories, whereas matter consists of extended systems with collective features. Combining the elementary-interaction aspect of high-energy physics with the macroscopic-matter aspect of nuclear physics, the subject of heavy-ion collisions is QCD thermodynamics, i.e. the study of bulk matter consisting of strongly interacting particles (hadrons/partons). The formalism to be used would ideally be the one of thermodynamics, where complex multi-particle states are described in terms of a few macroscopic variables.

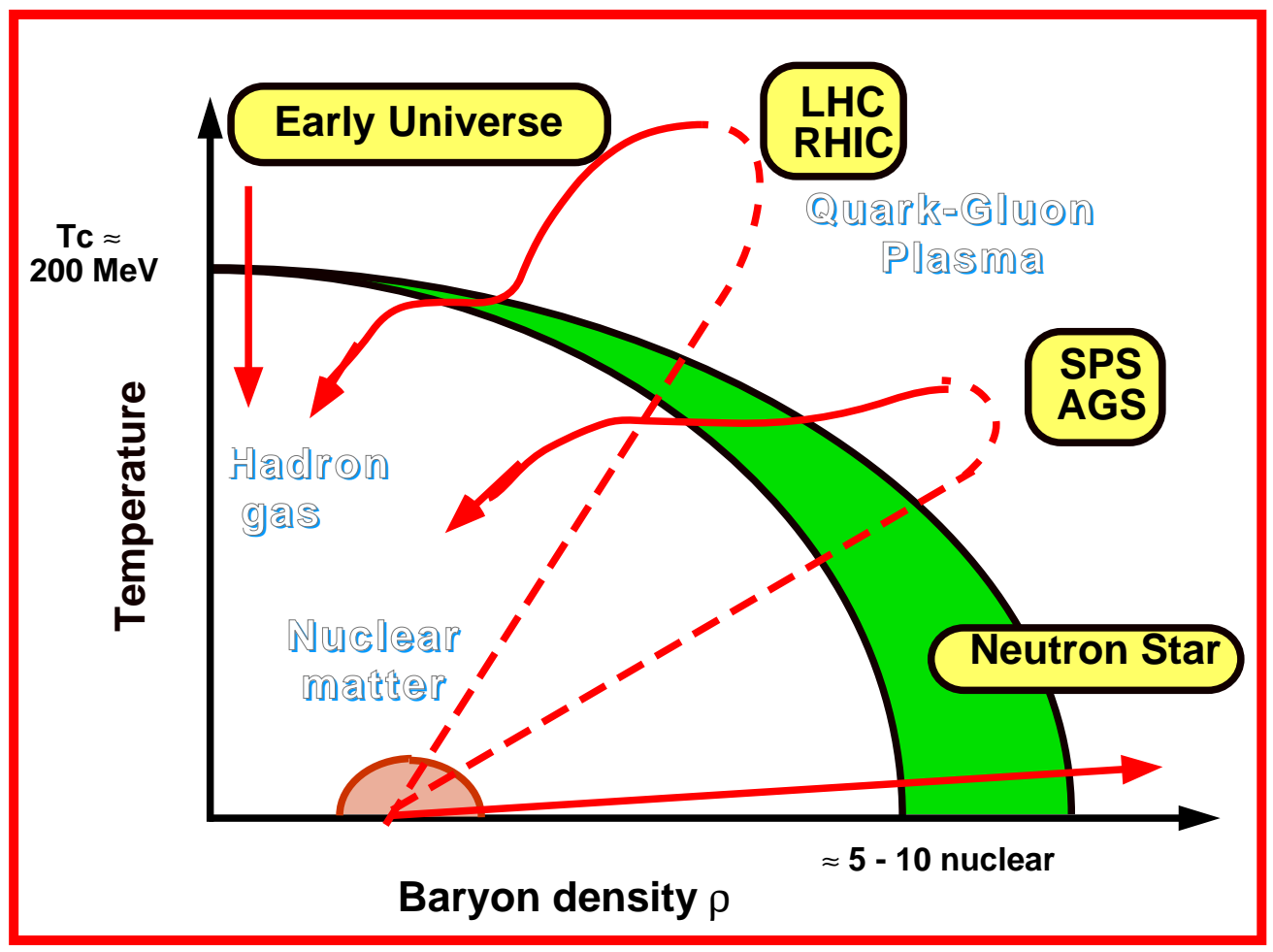

Figure 1: The phase diagram of hadronic matter and the hadron gas - quark-gluon plasma phase transition

This study is of interest to explore and test QCD on its natural scale $\left(\Lambda_{Q C D}\right)$ and to address the fundamental questions of confinement and chiral-symmetry breaking, which are connected to the existence and properties of the quark-gluon plasma. Moreover, it is of general relevance in understanding the dynamical nature of phase transitions involving elementary quantum fields, as the QCD phase transition is the only one accessible to laboratory experiments. 


\section{Current status and results}

\subsection{Initial conditions and global features}

The predictions of lattice QCD are rather firm in that a transition to the QGP should exist in the vicinity of a critical temperature $\mathrm{T}_{c}$ of $\approx 150-200 \mathrm{MeV}$ (whether the transition is of first order, second order, or only 'rapid' is still a matter of debate). However, whether the QGP is actually created in heavy-ion collisions at current energies is a different question and will depend on the dynamics of the reactions and in particular on the initial conditions of the system shortly after the collision. In order to reach the QGP, or even only to use macroscopic concepts (such as 'phase transition') and the language and variables of thermodynamics (such as 'temperature' or 'density'), the system has to be extended - i.e. its dimensions ought to be much larger than the typical scale of strong interactions - it has to be in (or near) equilibrium - i.e. its lifetime has to be larger than the relevant relaxation times - and the energy density $\epsilon$ has to exceed the critical threshold for QGP formation. This threshold is predicted by lattice QCD to be of the order of $1-3 \mathrm{GeV} / \mathrm{fm}^{3}$, equivalent to a temperature $T_{c}$ of $150-200 \mathrm{MeV}$ or a baryon density $\rho_{c}$ of 5 to 10 times normal nuclear matter density (see Fig. 1).

Present results from the ongoing fixed-target program indicate that the initial conditions realized in these reactions could indeed be favourable for QGP formation. In head-on central collisions, hundreds of particles are produced per unit of rapidity, the system expands to a size of the order of $1000 \mathrm{fm}^{3}$ (as measured by particle interferometry), and initial energy densities are estimated to exceed $2 \mathrm{GeV} / \mathrm{fm}^{3}$. However, the expansion is also extremely fast, with an estimated total lifetime of only a few fm/c from the first instance of the collision until the final freeze-out of hadrons.

While these results show that we are certainly close to the requirements listed above for QGP formation, they are by no means sufficient. In particular the energy density estimates are inversely proportional to the assumed 'formation time', i.e. the time needed to reach thermal equilibrium, and might well be smaller (or bigger ?) by a factor of the order of two. Also the lifetime of the system seems marginal, and even if a QGP is formed it might simply not live long enough for its signals to clearly stand out from the background created in later, hadronic phases of the evolution. The existance of a QGP phase can only be settled experimentally by searching for direct and specific signals.

\subsection{Recent experimental highlights}

In the following, I will concentrate on three main topics which are at the heart of the quest for the QGP, and in which significant progress has been achieved over the last two years, i.e. are there experimental indications for equilibrium hadronic matter, chiral symmetry restoration, and deconfiment?

Equilibrium hadronic matter ? While in principle the study of non-equilibrium hadronic matter might be of considerable interest, in practice the huge number of largely unknown dynamical parameters governing the evolution would today make the analysis of a complex system such as heavy ion reactions very difficult. The powerfull laws of thermodynamics can reduce this complexity and make definite and testable predictions, largely independent of the microscopic dynamics, for those degrees of freedom which evolve in equilibrium. The price to pay is a loss of information concerning events preceeding the equilibrium, as the memory of earlier (and possibly more interesting) stages of the evolution is largely lost.

In reality, we will have to deal with a hierarchy of processes and scales, some of which have large cross sections and correspondingly small relaxation times and therefore might 


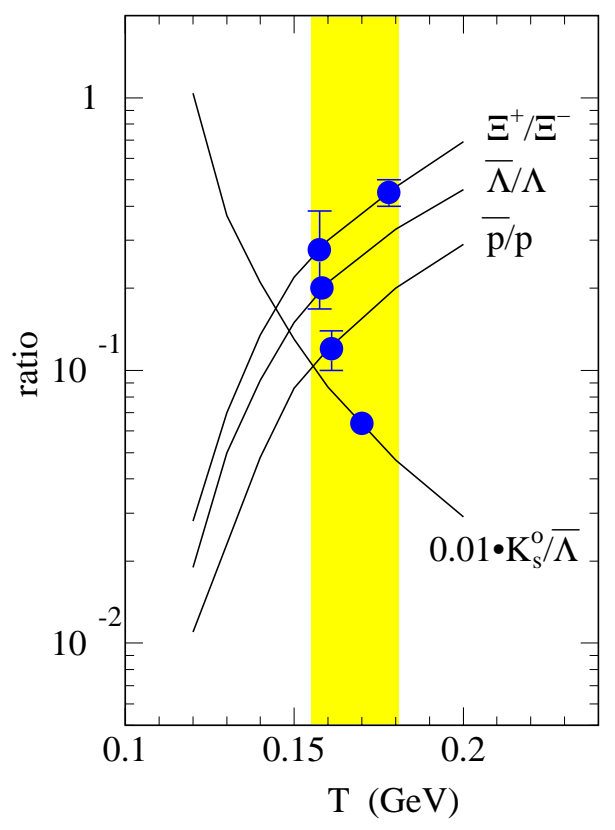

Figure 2: Hadrochemical equilibrium model calculation of hadron yields (full lines) which show a particularly strong sensitivity to the temperature of the system. In comparison are shown various experimental points from SPS experiments NA35, NA36, NA44, and WA85.
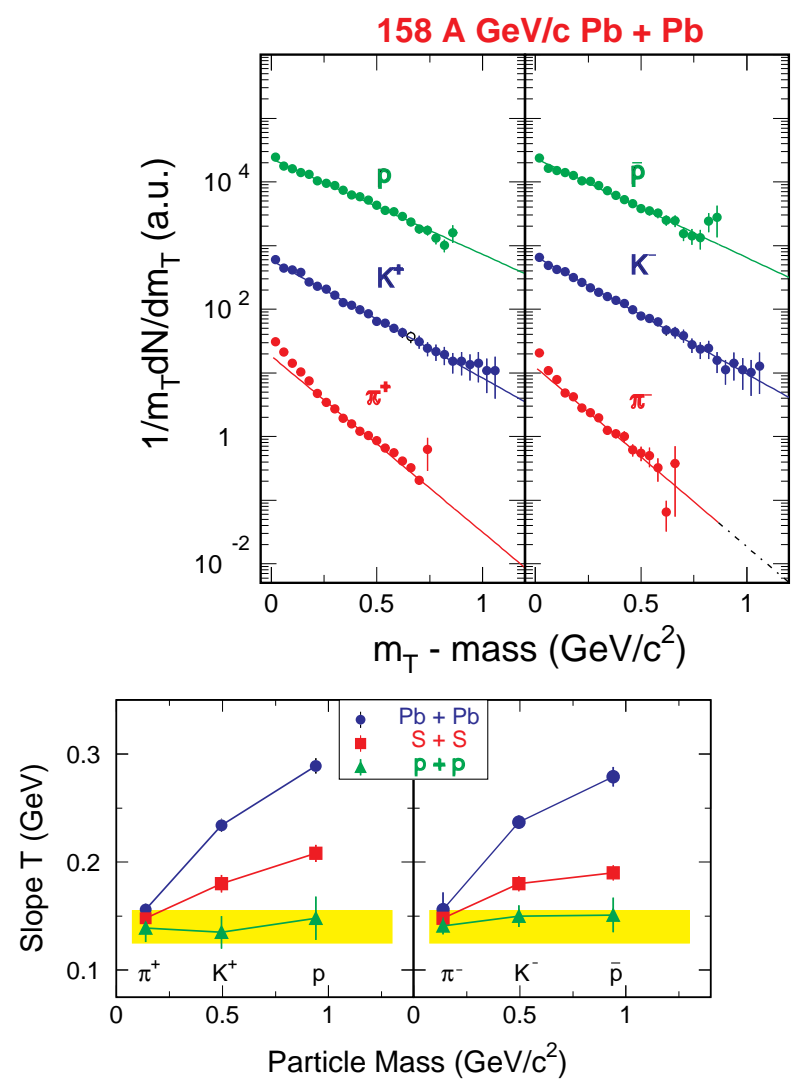

Figure 3: Transverse mass spectra (top) and inverse slope parameters (bottom) of pions, kaons and protons near midrapidity from NA44.

evolve close to equilibrium, and others which decouple early from a thermal evolution and are sensitive to the hot initial phase of the reaction. Prime candidates for the former are hadronic observables, like momentum spectra and particle ratios, and for the latter hard probes and electromagnetic signals.

In a purely thermal system of hadrons, the momentum distributions, when expressed as a function of the transverse mass $m_{T}\left(m_{T}=\sqrt{m^{2}+p_{T}^{2}}\right)$, will be independent of the particle mass with a slope inversely proportional to the temperature T. In an expanding system, an additional collective flow component can develop which blue-shifts the momentum spectra with a common transverse velocity $\beta_{T}$ leading to a mass dependent component. Likewise, the abundance of particle species in equilibrium hadronic matter is given by two independent parameters, i.e. the temperature $\mathrm{T}$ and a baryochemical potential $\mu_{B}$ (which reflects the baryon assymetry in the initial state). A hadronic system in both 'thermal' (momentum) and 'chemical' (particle abundances) equilibrium is therefore fully determined by only three independent parameters: T, $\beta_{T}$ and $\mu_{B}$.

Such a simple prescription seems to be indeed borne out by the data. This is illustrated in Fig. 2, which shows a comparison of measured particle ratios with predictions based on chemical equilibrium [1]. Within the experimental accuracy, these ratios as well as a large number of other particle abundancies are in rather good agreement for a temperature of about $160 \mathrm{MeV}$ and a baryon chemical potential of $\approx 180 \mathrm{MeV}$, corresponding 
to $1 / 3$ nuclear matter density (at the AGS the best fit values are $\mathrm{T} \approx 140 \mathrm{MeV}$ and $\mu_{b} \approx$ $500 \mathrm{MeV})$.

Taking this temperature value from particle ratios, momentum spectra of different particles in $\mathrm{Pb}-\mathrm{Pb}$ reactions [2] are well described if in addition a common flow velocity of $\beta_{T} \approx 0.4 \mathrm{c}$ is introduced (see right hand side of Fig.3). On the left hand side of Fig.3, the inverse $m_{T}$-slopes are shown for $\mathrm{pp}, \mathrm{S}+\mathrm{S}$ and $\mathrm{Pb}+\mathrm{Pb}$ reactions at comparable energies $(\sqrt{s} \approx 20 \mathrm{GeV})$. While the slopes in pp reactions are independent of particle type, i.e. exhibit ' $m_{T}$-scaling', the slope parameter increases proportional to the particle mass for heavier reaction systems.

A large set of independent hadronic observables, i.e. momentum spectra, particle ratios and HBT correlation results (which are are also sensitive to $\mathrm{T}$ and $\beta_{T}$ ), seems to be consistent with a suprisingly simple picture of the late stages of heavy ion reactions: a dense hadronic system expanding in almost complete thermodynamical equilibrium untill a sudden freeze-out fixes momentum spectra and particle ratios to the finally observed values. In addition, the location of this freeze-out point in the temperature-density plane is located very close to the phase boundary as sketched in Fig. 1 [1].

However, before this intuitively appealing scenario can be taken as established, a number of experimental and conceptual questions will have to be clarified. On the experimental side, better statistics (in particular for Hyperons) and large acceptance data will be needed to come to a more quantitative test of predictions. Competing models [3], which try to explain the flow-like behaviour seen in the momentum spectra by initial state scattering, have to be confronted with experiment. On the conceptual side, the most puzzling observation is that already very elementary reactions look practically as 'thermal' as heavy ion reactions. While it has been known, but never 'understood', that momentum spectra in hadronic reactions look 'thermal' (obey $m_{T}$-scaling), a recent re-analysis of pp and $\mathrm{e}^{+} \mathrm{e}^{-}$reactions has shown that also the particle ratios can be extremely well described with thermodynamics [4]. How can this be possible in systems containing a few hadrons only?

Assuming that some satisfactory answers to these questions can eventually be found, we could then go on to analyse the hadronic data in more detail to look for information on the dynamics preceeding the freeze-out. Relaxation times in a partonic and a hadronic medium are likely to be different, and therefore the question how and how fast did the system reach equilibrium in different channels are of interest, particular in the Hyperon sector, where hadronic relaxation times are suspected to be extremely long. Flow patterns should be sensitive to the equation of state of matter and therefore contain indirect evidence for a QGP phase transition preceeding freeze-out. And finally, single event analysis, which has barely started to give sensitive results, might still have some surprises in store. Chiral symmetry restoration ? Weakly interacting electromagnetic probes (photons or leptons) are a direct means of gaining information on the early dense and hot stages of the collision, as they leave the interaction volume without being altered by final state effects. While so far only upper limits exist for direct (thermal) photon production, recent data on lepton pairs show an unexpectedly large yield at low masses below the $\varrho$ meson.

Figure 4 shows the electron pair mass spectrum observed in central $\mathrm{S}+\mathrm{Au}$ collisions by NA45 [5]. The upper part summarizes model calculations which include contributions from hadronic decays (shaded area) and from in-medium pion annihilation and bremsstrahlung. An excess at $0.2<m\left(e^{+} e^{-}\right)<0.6$ remains unexplained. The lower panel exhibits perfect agreement with the data obtained in models which inlcude in addition an in-medium modification of the $\varrho$ and $\omega$ masses. A similar excess, consistent with the 

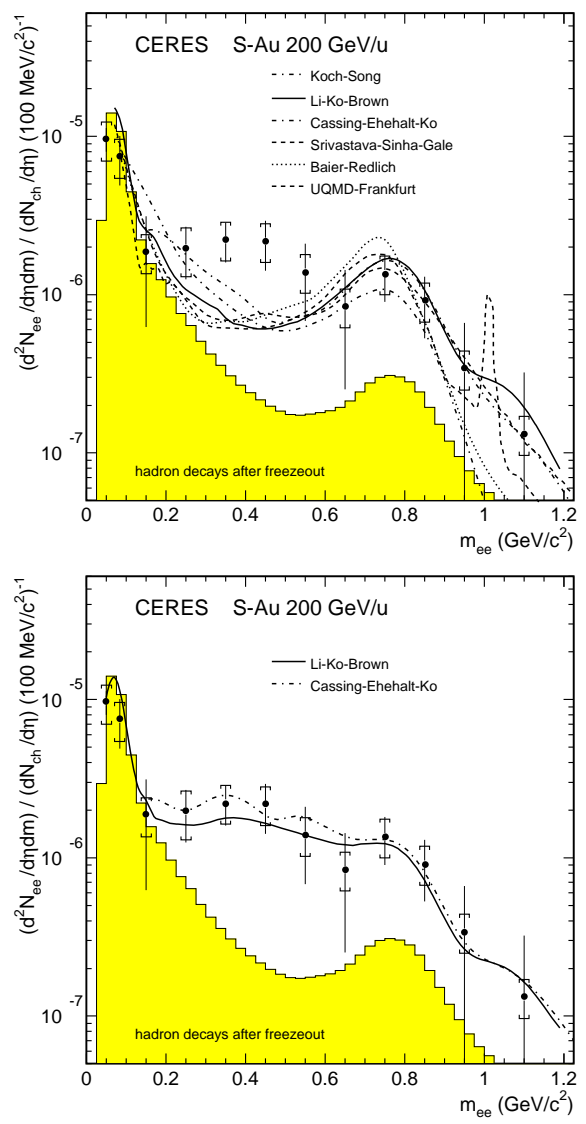

Figure 4: Di-electron invariant mass distribution measured by the NA45 experiment in central $\mathrm{S}+\mathrm{Au}$ collisions, compared to calculations including hadronic decays and effects expected for high pion densities (top) and calculations incorporating in addition a density dependent mass shift of the $\varrho$ meson (bottom).

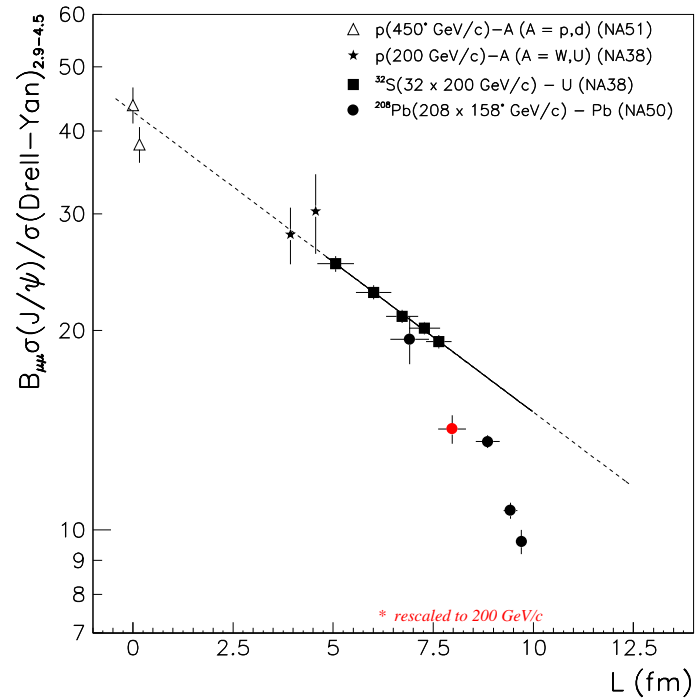

Figure 5: $J / \Psi$ production for proton, sulphur and lead induced collisions relative to the Drell-Yan yield as a function of the thickness $\mathrm{L}$ of matter traversed on average. All data except central $\mathrm{Pb}+\mathrm{Pb}$ are consistent with a suppression which is exponential in $\mathrm{L}$.

same model calculation, has been found in the $\mu^{+} \mu^{-}$mass spectrum by NA34/3 [5].

In-medium modification of vector mesons, if experimentally confirmed by more conclusive data, could be a direct consequence of the chiral symmetry transition at the phase boundary between hadronic matter and the QGP. The rapidly varying quark condensate should lead to changes in the properties of hadrons (masses, width) in the vicinity of the phase transition, which will be observable in the lepton mass spectrum for mesons decaying in the dense transition regime. This would indeed be a spectacular verification of the concept underlying the generation of light hadron masses in QCD.

An excess in the intermediate mass range $(1.5-2.5 \mathrm{GeV})$ observed in muon pairs by NA50 has so far not found any convincing interpretation [6].

Deconfinement ? Signals originating from hard-scattering processes at the very beginning of the reaction are an ideal tool to probe the state of the surrounding QCD matter. The original idea [7] that $\mathrm{J} / \Psi$ production should be suppressed in a QGP relies on a 
Debye screening mechanism which renders colour interactions short ranged in a dense medium ('deconfinement') and therefore prevents the formation of bound resonances.

$\mathrm{J} / \Psi$ suppression with about the characteristics as predicted for a QGP was indeed one of the first results reported from heavy ion experiments in 1987. Its subsequent interpretation, alternating repeatedly between 'trivial' and 'exciting', is probably the best example on how our undestanding of nuclear collisions has progressed in a constant interplay between theory and experiment, new explanations and new data. An up-to-date compilation of $\mathrm{J} / \Psi$ production relative to the Drell-Yan continuum in $\mathrm{pA}$ and $\mathrm{AB}$ reactions is shown in Fig. 5 versus the average path length $\mathrm{L}$ traversed by the $c \bar{c}$ pair after its creation inside the target and projectile nuclei [8]. Up to and including central S-U collisions, this ratio decreases exponentially with L, consistent with a nuclear absorption cross section of $6 \mathrm{mb}$. It took the better part of the last ten years, a variety of data for $\mathrm{J} / \Psi, \Psi^{\prime}$ and $\Upsilon$ - from low energy pp and $\mathrm{pA}$ reactions to photoproduction and high $p_{T}$ production at the Tevatron - and a good measure of other ingredients (nuclear structure functions, initial and final state scattering, formation time, ..) to come to a consistent and theoretically substantiated interpretation $[9,10]$. The exponetial attenuation is today seen as resulting from the interaction between the nuclear medium and a pre-resonance state, a coloured $c \bar{c}$-gluon configuration which evolves only later (and outside the nucleus) after some finite formation time into the physical, colour neutral $\mathrm{J} / \Psi$ or $\Psi$ ' hadron. So $\mathrm{J} / \Psi$ suppression has provided a lot of insight into the dynamics of charmonium production, hadron formation and using the nucleus as a tool to measure short time scales, with no room left for QGP effects.

The extrapolation of this model to central $\mathrm{Pb}-\mathrm{Pb}$ reactions was straight forward, essentially parameter free, and completely wrong (see Fig. 5) ! The Pb-Pb data, wether plotted as function of L or any other variable, shows significantly less $\mathrm{J} / \Psi$ 's than hadronic absorption models would predict by extrapolating fom light ion and pA results. While some debate still persists if the additional suppression is really 'anomalous' or not, new precision data which is currently analysed should settle this question in the near future. Most likely, some additional physics will have to be included in order to describe the $\mathrm{Pb}$ data. However, wether this 'new physics' will require deconfimenemt, dynamical pre-curser phenomena of the QGP transition, or just some overlooked hadronic effect, remains to be seen.

\subsection{Future fixed target program}

Given the recent exciting developments, but also their rather preliminary character, the future directions are perfectly clear. The current SPS fixed target program is unique in the world, it adresses a well focused set of fundamental questions, it has entered an extremly productive phase and it has now to be brought to its full potential. With the exception of the Hyperon and the low mass lepton pair measurements, statistics is in general not a problem. Rather the future program has to provide the answers to some well identified questions: An inverse kinematic experiment - shooting a $\mathrm{Pb}$ beam on a proton target- will give important input concerning $J / \Psi$ interactions with confined (nuclear) matter. By changing either the beam energy or projectile and target mass, the onset of anomalous $J / \Psi$ suppression should change in a characteristic way revealing the relevant scaling variable. With the same means (i.e. change in energy or projectile type), one can study the onset of strangness enhancement and the evolution of thermal variables in general and collective flow in particular. A run at the lowest possible SPS energy, around $40 \mathrm{GeV} /$ nucleon, will increase the baryon density, possibly close to its maximum 
value. Signals related to chiral symmetry restoration, in particular the low mass lepton pairs, will in general be rather sensitive to baryon density. The low energy run can also make contact with the AGS regime and will allow the CERN experiments, which are quite distinct in their capabilities from the AGS detectors, to compare with and complement the program at lower energies. This program will require running for about five more years untill at least about 2001 and promises an intense and productive time untill, around the turn of this century, the new generation of heavy ion collider will come into operation.

\section{$3 \quad$ Heavy ion physics of the next century}

The study of ultra-relativistic heavy-ion collisions is a rather new, but rapidly evolving field. After the pioneering experiments at the BEVALAC and in DUBNA with relativistic heavy ions $(E / m \approx 1)$, the first experiments started in 1986 with light ions almost simultaneously in Brookhaven (AGS) and at CERN (SPS). Really heavy ions $(\mathrm{A} \approx 200)$ are available in the AGS since the end of 1992 and at the SPS since the end of 1994. When the colliders RHIC $(\sqrt{s}=200 \mathrm{GeV} / \mathrm{n})$ and LHC $(\sqrt{s}=5.5 \mathrm{TeV} / \mathrm{n})$ come into operation in 1999 and 2005, respectively, the available energy in the centre-of-mass will have increased by almost five orders of magnitude within 20 years. This unprecedented pace was made possible only by (re)using accelerators, and to some extent even detectors, built over a much longer time scale for use in high-energy physics. The following sections will summarize the physics and experiments to come in these latest (and possible last) heavy-ion machines.

Heavy ion collisions at the colliders are expected to provide a qualitatively different environment from existing accelerators by creating a very hot, and therefore more clearly detectable QGP via hard initial parton scatterings that can be calculated rather precisely. Extrapolating from present results to LHC, all parameters relevant to the formation of the QGP will be more favourable: the energy density, the size and lifetime of the system, and relaxation times should all improve by a large factor, typically by an order of magnitude compared to $\mathrm{Pb}+\mathrm{Pb}$ collisions at the SPS. We expect particle densities of several thousand per unit of rapidity, a freeze-out volume approaching $100,000 \mathrm{fm}^{3}$, and an initial energy density orders of magnitude larger than the one of normal nuclear matter. The initial temperature might be close to $1000 \mathrm{MeV}$, as compared to a value of $400-500 \mathrm{MeV}$ at RHIC and about $200 \mathrm{MeV}$ at the SPS. The energy densities and temperatures at LHC should be far above the deconfinement threshold, allowing us to probe the QGP in its asymptotically free "ideal gas" form.

The analysis of extended strongly interacting matter at both colliders will move from the fixed target regime dominated by soft phenomena into a domain where hard interaction between the primary partons will lead to a rapid production of further partons, and the interactions within this dense partonic medium, with the resulting strong increase in entropy, are expected to produce the QGP. The abundant formation of "minijets" with transverse momenta of a few $\mathrm{GeV}$ plays an essential role in this process. Perturbative QCD calculations can be used to construct and evaluate such parton interaction cascades; they indeed show the expected rapid rise towards thermalisation, on time scales considerably below $1 \mathrm{fm} / \mathrm{c}$, to the extremly high initial temperatures mentioned.

In order to verify that a QGP was produced and to study its properties, we need probes sensitive to the earliest and hottest stages of the medium. Three such probes are currently known: Bound heavy quark resonances (quarkonia), hard jets, and thermal dileptons/photons. Only charmonia as deconfinement probes have been studied at the SPS as discussed above; for all others, higher incident energies appear necessary. 


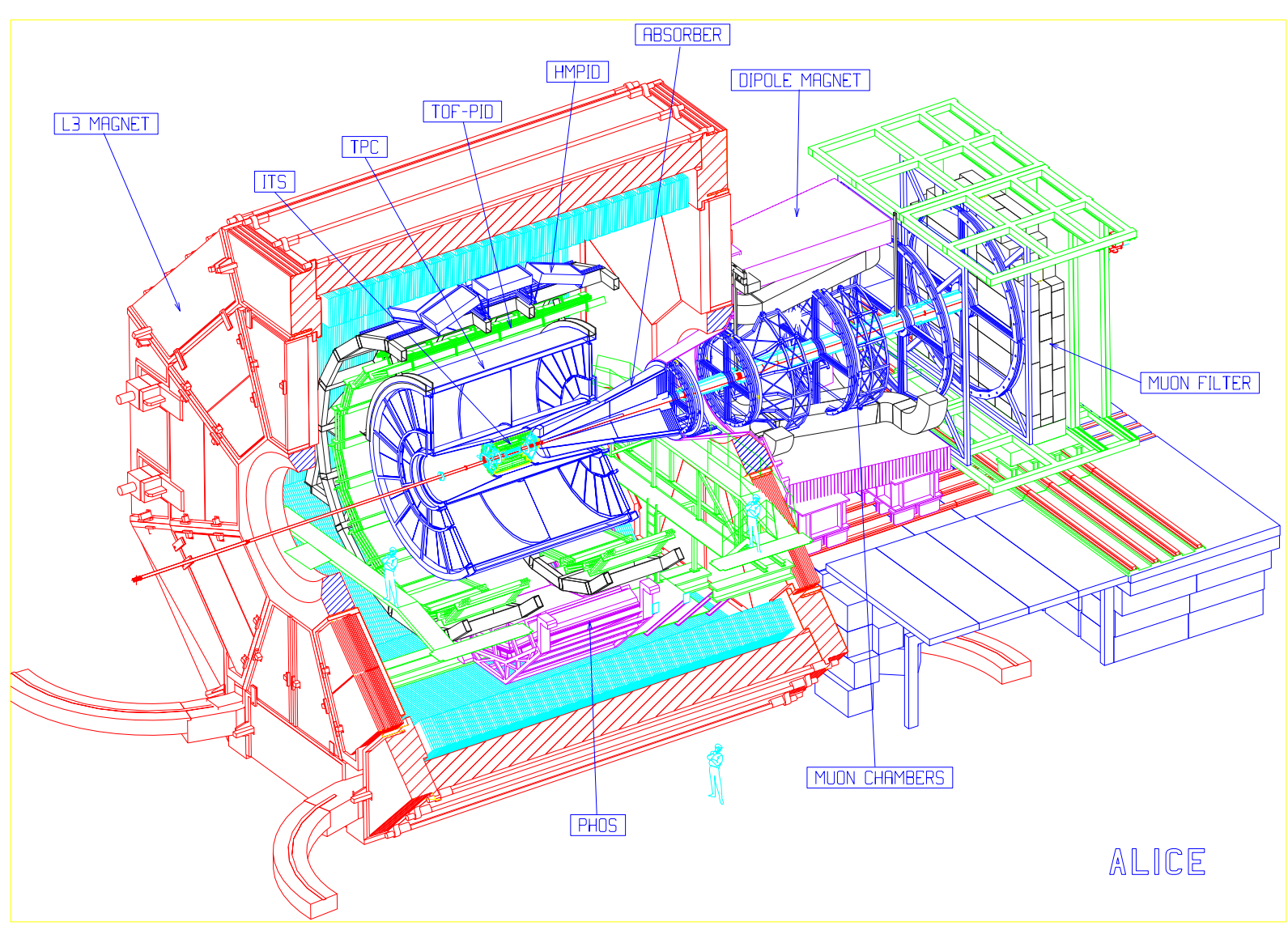

Figure 6: Schematic view of the ALICE detector which combines a central barrel for electron pair, photon, and multihadron studies, with a forward dimuon spectrometer.

To complete the deconfinement analysis of hot and dense matter, it must be extended to bottonium states. The $\Upsilon$, with its very small radius, can be dissociated only at the highest energy density attainable at LHC (of order $30 \mathrm{GeV} / \mathrm{fm}^{3}$ ), while the excited states $\Upsilon^{\prime}$ and $\Upsilon^{\prime \prime}$ are comparable to the charmonium resonances and will serve as important consistency checks.

Hard jets probe the produced medium through the energy loss of partons passing through dense matter. The theoretical aspects of this problem were recently studied in considerable detail. In particular, the rate of energy loss was found to depend quite sensitively on the size of the medium and there now are indications that the energy loss in cold nuclear matter is much smaller than that in a hot QGP. The production and subsequent attenuation of fast partons will add a crucial new penetrating probe to diagnose the nature of the strongly interacting matter produced in heavy ion collisions.

The temperature of the primordial medium could be best determined through measurement of the spectra of real or virtual photons. The thermal photon spectrum will be an integral over the temperature history of the system. Superimposed are the soft photons from the late hadronic stage as well as the primary Drell-Yan or hard QCD photons. Whether there is a window in transverse momentum (around one to a few $\mathrm{GeV}$ ) to actually measure such thermal dileptons or photons depends crucially on the density of the produced system; fortunately, conditions could be quite favourable at the high energy densities predicted at RHIC and LHC.

Another unique feature of heavy-ion collisions at the colliders is the possibility to 
measure a large number of observables with very good accuracy on an event-by-event basis: impact parameter, multiplicity, particle ratios and spectra and, of particular importance, size and lifetime from interferometry. Single event analysis, currently pioneered by NA49 at the SPS, will become a precision tool at very high multiplicity. One of the important design considerations for both the STAR detector at RHIC and the ALICE detector at LHC (Fig. refalice) is to make full use of this opportunity. It will allow the study of correlations and non-statistical fluctuations which would otherwise be washed out when averaging over many events. Such fluctuations are, in general, associated with critical phenomena in the vicinity of a phase transition.

\section{Summary}

The still very young field of ultra-relativistic heavy ion physics has proceeded since its inception in 1986 through three essential phases:

The initial round of 'exploratory' experiments has shown that appropriate detectors and analysis procedures can cope with the extreme particle densities produced in heavy ion collisions. They have qualitatively shown that an extended, interacting and very dense system has been formed that differs in many observables from the more elementary hadron-hadron reactions investigated in the past. Falling short of striking discoveries, this phase has nevertheless provided a 'principle proof of feasibility' and has substantiated the expectation that heavy ion collisions are an appropriate tool to create equilibrium hadron matter and eventually the quark-gluon plasma.

The next phase was characterized by efforts to get a comprehensive and precise set of data and to come to a quantitative understanding of the experimental results. A close and very effective interaction between theory and experiment, models and data, has led to significant progress in identifying relevant ingredients and important microscopic processes.

The field is currently in its third, and most dramatic phase. Results from both AGS and SPS with real 'heavy' ion beams have produced puzzling results which strongly hint at a picture of high energy nuclear reactions almost to good to be true: A pre-mordial phase of deconfined partons - the QGP ? - responsible for quarkonium suppression, followed by a transition regime with gradual onset of chiral symmetry breaking leading to changes in the properties of light hadrons, concluded by a gas of hadronic matter governed by the simple laws of thermodynamics. On the short term, the ongoing experiments should provide us with additional and more complete data in order to substantiate (or refute) this scenario.

On the longer term, making use of RHIC and LHC for heavy ion collisions provides an unparalled opportunity for exploring the physics of QCD matter in a qualitatively very different and unique region of extremely high energy density. RHIC and its four major experiments (STAR, PHENIX, BRAHMS and PHOBOS) will make the first step, starting in 1999 with $\mathrm{Au}+\mathrm{Au}$ collisions an order of magnitude above what is currently available at the SPS. The LHC, with a centre-of-mass energy almost thirty times above RHIC, will lead by 2005 into a region comparable only to the highest energy cosmic ray events. Its single dedicated detector, the ALICE experiment, has recently (February 1997) been approved and it represents the long term future of the ultra-relativistc heavy ion program. Building a detector of the size and complexity required for LHC will be an unprecedented challange, and its sucessfull completion will need the continued, strong and emphatic support and participation of the heavy ion community worlwide. 


\section{References}

[1] P. Braun-Munzinger, J. Stachel, H. Wessels, N. Xu, Phys Lett. B 366 (1996) 1 and J. Stachel, Nucl. Phys A610 (1996) 509c.

[2] N. Xu et al, Nucl. Phys A610 (1996) 175c.

[3] A. Leonidov, M. Nardi, H. Satz, Nucl. Phys A610 (1996) 124c.

[4] F. Becattini, U. Heinz, preprint DFF 268/02/1997 (subm. Zeit. Phys. C).

[5] A. Drees et al, Nucl. Phys A610 (1996) 536c.

[6] E. Scomparin et al, Nucl. Phys A610 (1996) 331c.

[7] T. Matsui, H. Satz, Phys. Lett. B 178 (1986) 416.

[8] M. Gonin et al, Nucl. Phys A610 (1996) 404c.

[9] C. Lourenco, Nucl. Phys A610 (1996) 552c.

[10] D. Kharzeev, Nucl. Phys A610 (1996) 418c. 